\title{
Synthesis and ionic conductivity of mechanically synthesized yttrium-doped ceria solid solutions
}

\begin{abstract}
Doped ceria is considered the most promising high-conducting electrolyte, alternative to the commercially used yttria-stabilized zirconia. The ceria fluorite structure is very tolerant to dissolution of lower valent metal ions. The present employed mechanical method, introduces comparison of mechanical-synthesized yttrium-doped ceria system, denoted as Ce1-xYxO2-ŭ (0.05ÒxÒ.4), which were successfully synthesized by both conventional solid-state and mechanochemical methods. In mechanochemical reaction, fine-grained powders with uniform grain size distribution were obtained. X-ray diffraction analysis showed all solid solutions were single phase with cubic fluorite structure. The electrical conductivity of sintered samples of calcium doped ceria compounds were investigated in air as a function of temperature using AC impedance spectroscopy. Impedance data showed that yttrium-doped ceria electrolyte is a good ionic conductor with conductivity as high as 10-3 ohm cm-1. Further characterization using Differential Thermal Analysis (DTA), Thermo Gravimetric Analysis (TGA) and Scanning Electron Microscopy (SEM) were carried out.
\end{abstract}

Keyword: Oxide ion conductor; Yttrium; Ceria 